\title{
COMPACTNESS OF CONFORMAL METRICS WITH INTEGRAL BOUNDS ON CURVATURE
}

\author{
Thesis by \\ Matthew J. Gursky \\ In Partial Fulfillment of the Requirements \\ for the Degree of \\ Doctor of Philosophy
}

California Institute of Technology

Pasadena, California

1991

(Submitted May 23, 1991) 


\section{Acknowledgments}

In the acknowledgments section it is a common practice to dutifully thank one's adviser for their "helpful comments and suggestions". My indebtedness to Alice Chang runs much deeper: For her willingness to accept a student from a different school, her unwavering support through the inevitable difficult times in my thesis work, and yes, for her helpful comments and suggestions. I consider Paul Yang to be my co-adviser, and I am equally grateful for his time and patience in teaching me geometry.

Enduring graduate school would not have been possible without the support of my family and friends, especially my parents. I would also like to thank Fr. Brian Wilson and Fr. Thomas White for helping me to understand why mathematics is important.

Finally, I would like to dedicate this thesis to my wife, Susie, as a modest expression of my appreciation for all that she has done for me.

Laus Tibi, Christe. 


\begin{abstract}
In this thesis, we show that a sequence of conformal metrics on a compact $n$ dimensional Riemannian manifold ( $n \geq 4$ ) which has an upper bound on volume and an upper bound on the $L^{p}$ norm of the curvature tensor for fixed $p>n / 2$ has a subsequence which converges in $C^{\alpha}$. If $n=3$, we have the same result if we assume, in addition, that the scalar curvature has an $L^{2}$ bound.

As corollaries, we have the compactness of a sequence of conformal metrics on a compact three-manifold which are isospectral with respect to either the standard or conformal Laplacian, and the result of Lelong-Ferrand that any compact manifold with non-compact conformal group is conformally equivalent to the standard sphere.
\end{abstract}


Table of Contents

Acknowledgments $\ldots \ldots \ldots \ldots \ldots \ldots \ldots \ldots$ ii

Abstract $\ldots \ldots \ldots \ldots \ldots \ldots \ldots \ldots \ldots \ldots$ iii

Table of Contents $\ldots \ldots \ldots \ldots \ldots \ldots \ldots \ldots$ iv

Introduction $\ldots \ldots \ldots \ldots \ldots \ldots \ldots \ldots \ldots$

Section 1. Preliminaries ............. 6

Section 2. Analysis of the Blow-up ........ 8

Section 3. Finding an Upper Bound ........ 21

Section 4. Finding a Lower Bound ......... 34

Section 5. The Degenerate Case ......... 42 


\section{INTRODUCTION}

A natural question which arises in geometry, and has its beginnings in the very origin of the subject, is the relationship between the topological, geometric, and analytic properties of a given Riemannian manifold. The Gauss-Bonnet theorem is perhaps the best known example, demonstrating the connection between the topological and geometric properties of a compact surface in a rather precise way. However, there are many "pinching", "rigidity", "finiteness", and "compactness" results which seek to give conditions on the curvature (diameter, volume, etc.) that guarantee that any manifold with these properties belongs, for example, to a certain diffeomorphism class (in the case of a finiteness theorem) or to a compact family in some topology (in the case of a compactness theorem). While the conditions and conclusions of these results vary, they all make some assumption on the sign or size of the curvature, which can be said to be the central Riemannian invariant. Indeed, many of these results are formulations of the question, how does the curvature determine the metric?

Returning to the theory of surfaces, we have a very satisfying result: By the uniformization theorem, on any compact surface each metric is conformally equivalent to a "model" metric of constant curvature. Therefore, given a compact surface with a metric of, say, positive curvature, then we know that the surface is topologically the sphere and the metric is conformally equivalent to the standard round metric. In higher dimensions the situation is more complicated, but a brief survey of some of the known results will provide a nice background for the main theorem to be proved here. 
As an attempt to generalize the uniformization theorem, Yamabe $[\mathrm{Y}]$ asked whether a given compact Riemannian manifold of dimension three or greater is conformal to one of constant scalar curvature. His approach was to attempt to solve the elliptic equation satisfied by a conformal metric of constant scalar curvature using the methods of the calculus of variations. Although he claimed to have answered the question in the affirmative, N. Trudinger ([T]) found an error in Yamabe's proof which he was only able to fix if the Yamabe constant $Q\left(M, g_{0}\right) \leq 0$. The complete solution of the Yamabe problem, that is, the treatment of the case where $Q\left(M, g_{0}\right)>0$, took over sixteen years (for an exhaustive survey, see [LP]).

The difficulty of the problem lies in the conformal invariance of the equation to be solved and the fact that the conformal group of the standard sphere is non-compact. Therefore, a sequence of conformal metrics with fixed volume and scalar curvature approaching a constant may not converge. The key to solving the problem was finding a way to distinguish whether a given manifold was conformally equivalent to the standard sphere. The proof of the positive mass conjecture by Schoen and Yau provided such a criterion, and Schoen [S] used it to complete the solution of the Yamabe problem.

The goal of this thesis is to provide a compactness criterion for metrics in a fixed conformal class. In the Yamabe problem, the sequence of conformal metrics was a minimizing sequence for the scalar curvature functional; in our setting the sequence is only assumed to satisfy an integral bound on the curvature, but the same degeneracies may occur. Before we discuss this, however, let us try to justify the conditions we impose on the sequence in order to conclude compactness. 
In the uniformization theorem, the "model" metrics we discussed have constant curvature. In [OPS1], the authors gave another characterization of these metrics: Roughly speaking, they maximize the determinant of the Laplacian functional. Later ([OPS2]), they applied many of the techniques and results of [OPS1] to show that a sequence of conformal metrics on a closed compact surface which is isospectral with respect to the Laplacian are compact in the $C^{\infty}$ topology (modulo Moebius transformations). One can view this as a result connecting the analytic and geometric properties of a compact surface.

There are two ingredients in their proof. To begin with, the isospectral assumption, as is well known (see, for example [G], [MS]), implies bounds on certain integrals involving the curvature, i.e., "local" information. However, this local information is not sufficient to conclude compactness (for example, the main theorem of this thesis is not true in two dimensions, even if we allow a much stronger $L^{p}$ condition on the curvature). Hence some "global" invariant is needed, and the determinant of the Laplacian is used. In [BPY], [CY1], [CY2], the authors prove a similar compactness result in three dimensions for isospectral conformal metrics, and the first eigenvalue of the Laplacian provides the global invariant.

In this thesis, we are able to dispense with any global quantities - a somewhat surprising result in view of our comments above. A precise statement follows:

THEOREM. Let $\left(M, g_{0}\right)$ be a compact $n$-dimensional Riemannian manifold with $n \geq 3$. Let $\left\{g_{k}=u_{k}^{4 / n-2} g_{0}\right\}$ be a sequence of conformal metrics which satisfy

$$
\operatorname{Vol}\left(g_{k}\right)=\int u_{k}^{\frac{2 n}{n-2}} d V_{g_{0}} \leq V_{0}
$$




$$
\int\left|R m\left(g_{k}\right)\right|^{p} d V_{g_{k}} \leq \beta^{p},
$$

for some constants $V_{0}, \beta$ and $p>n / 2$ (here $\left|R m\left(g_{k}\right)\right|$ is the norm of the curvature tensor of $g_{k}$ ). If $n=3$, assume in addition that

$$
\int R_{k}^{2} d V_{g_{k}} \leq \tilde{\beta}^{2}
$$

where $R_{k}$ is the scalar curvature of $g_{k}$.

Then there are constants which depend on $V_{0}, \beta$, and $p$ such that

$$
\begin{gathered}
C_{1}^{-1} \leq u_{k} \leq C_{1} \\
\left\|u_{k}\right\|_{2, p} \leq C_{2}
\end{gathered}
$$

(unless $\left(M, g_{0}\right)$ is conformally equivalent to the sphere with the standard metric, in which case (0.3) and (0.4) hold modulo the conformal group, i.e., there is a sequence of conformal transformations of $M\left\{T_{k}\right\}$ such that if

$$
\nu_{k}^{4 / n-2} g_{0}=T_{k}^{*} g_{k}
$$

then (0.3) and (0.4) hold for $\left.\nu_{k}\right)$. Hence a subsequence of $\left\{u_{k}\right\}$ converges in $C^{\alpha}(M)$ with $0<\alpha<(2 p-n) / p$.

While other compactness results (see, for example, $[\mathrm{P}],[\mathrm{Yg}]$ ) have a more general setting, we make only minimal assumptions on the curvature and conclude a stronger type of convergence. 
In dimension three, assuming the sequence is isospectral with respect to either the standard or conformal Laplacian, then the heat invariants supply an $L^{2}$ curvature bound and we have as an immediate corollary:

COROLLARY A. A sequence of conformal metrics on a compact 3-manifold which are isospectral with respect to either the standard or conformal Laplacian is compact in $C^{\alpha}$ (modulo the conformal group).

In fact, it follows from the work in [BPY] and [G] that such sequences are compact in the $C^{\infty}$ topology, but we will not pursue this here.

Let $\left(M, g_{0}\right)$ be as in the main theorem. If $\Gamma$ denotes the conformal group of $\left(M, g_{0}\right)$, then if $\Gamma$ is not compact we may choose a sequence $\left\{\varphi_{k}\right\} \subset \Gamma$ which does not have a convergent subsequence and consider the metrics $g_{k}=u_{k}^{4 / n-2} g_{0}=$ $\varphi_{k}^{*} g_{0}$. This sequence certainly satisfies the hypotheses of the main theorem (each $g_{k}$ is isometric to $\left.g_{0}\right)$. It follows from the results of $\S 3$ and $\S 4$ that $Q\left(M, g_{0}\right)>0$ and $\left(M, g_{0}\right)$ is locally conformally flat. Hence, by the results of $\S 5$ (which extend the work of [CY2]), we have as a corollary the following result of Lelong-Ferrand $([\mathrm{LF}])$ :

COROLlARY B. A compact $n$-dimensional manifold $\left(M, g_{0}\right)$ has non-compact conformal group if and only if $\left(M, g_{0}\right)$ is conformally equivalent to the standard sphere.

We now give a brief outline of the proof of the main theorem. In Section 2, we show that if the sequence $\left\{u_{k}\right\}$ does not have a uniform upper bound, then it blows up at finitely many points, and off these blow-up points we may extract a 
subsequence which converges uniformly on compacta. Hence $\left\{u_{k}\right\}$ has an almost everywhere defined limit which we denote by $w$, and we conclude the section by showing that $w$ is in fact bounded above (a fact which will be useful later).

In Section 3 (which is the heart of the argument) we prove a kind of Harnack estimate: That is, if $\left\{u_{k}\right\}$ has a positive uniform lower bound, then it has an upper bound. Hence the remainder of the paper is devoted to finding a lower bound.

To this end, in Section 4 we show that in case the Yamabe constant $Q\left(M, g_{0}\right)=$ $Q \leq 0$, then $\left\{u_{k}\right\}$ has a lower bound. Also, if $M$ is not locally conformally flat, then a lower bound is easily achieved. We also show that if $\left(M, g_{0}\right)$ is conformally equivalent to the sphere with the standard metric, then (modulo the conformal group) $\left\{u_{k}\right\}$ is bounded below. This leaves the case where $Q>0$ and $M$ is locally conformally flat.

In Section 5 we imitate the argument of [CY2] and show that if $Q>0$ and $\left\{u_{k}\right\}$ does not have a lower bound, then by rescaling off the blow-up points we can build a complete, flat metric on $M$ and show that $M$ is in fact conformally equivalent to the sphere.

\section{$\S 1$. Preliminaries}

We begin by establishing some notation and recalling some basic results from geometry. If $(M, g)$ is a Riemannian manifold, then $R$ (or $R_{g}$ if there is the possibility of confusion) will denote the scalar curvature, $R c\left(R c_{g}\right)$ the Ricci 
tensor, and $R m\left(R m_{g}\right)$ the full curvature tensor. Of course $|R| \leq|R c| \leq|R m|$, where in the last two expressions the norm of the tensor is meant. $\nabla_{g}$ will denote the gradient vector field in the $g$ metric, but $\nabla$ will usually denote $\nabla_{g_{0}}$ to simplify notation. Likewise $d V_{g}$ will denote the volume form and $d V_{0}=d V_{g_{0}} . \Delta_{g}$ will denote the Laplace-Beltrami operator, and $L_{g}=\Delta_{g}-\alpha_{n} R_{g}$ the conformal Laplacian (throughout our work, $\left.\alpha_{n}=(n-2) / 4(n-1)\right) .\left|\nabla_{g}^{2} \varphi\right|$ will denote the norm of the Hessian tensor in the $g$ metric.

If $u$ is a smooth positive function on $M$ and $h=u^{4 / n-2} g$ is a conformal change of metric, then

$$
R_{h}=-\alpha_{n}^{-1}\left(L_{g} u\right) u^{-N+1}
$$

where $N=2 n / n-2$. This implies the "conformal covariance" of $L$ :

$$
L_{h} \varphi=u^{-N+1} L_{g}(u \varphi) .
$$

$Q\left(M, g_{0}\right)$ will denote the Yamabe constant; i.e.,

$$
Q\left(M, g_{0}\right)=\inf _{\varphi \neq 0} \frac{E_{g_{0}}(\varphi)}{\left(\int|\varphi|^{N} d V_{0}\right)^{2 / N}}
$$

where $E$ is the Dirichlet energy with respect to $g_{0}$ :

$$
E_{g_{0}}(\varphi)=\int\left|\nabla_{g_{0}} \varphi\right|^{2} d V_{0}+\alpha_{n} \int R_{g_{0}} \varphi^{2} d V_{0}
$$

$E$ is conformally invariant in the sense that if $h=u^{4 / n-2} g$, then $E_{h}(\varphi)=$ $E_{g}(u \varphi)$. This implies that $Q\left(M, g_{0}\right)$ is a conformal invariant: $Q\left(M, g_{0}\right)=$ $Q\left(M, u^{4 / m-2} g_{0}\right)$, so to simplify notation we usually just write $Q$ (since we are 
working in a fixed conformal class, there is no ambiguity). After a conformal change if necessary, we will assume throughout that $R_{0}=R_{g_{0}}$ has constant sign, and that sign is the same as the sign of $Q$.

The Sobolev constant of $g_{0}$ will be denoted by $A_{0}$ :

$$
\left(\int|\varphi|^{N} d V_{0}\right)^{2 / N} \leq A_{0}\left[\int|\nabla \varphi|^{2} d V_{0}+\int \varphi^{2} d V_{0}\right]
$$

Given $x \in M, \rho>0, B(x, \rho)$ will denote the geodesic ball of radius $\rho$ centered at $x$ (in the $g_{0}$ metric). For fixed $x \in M$ we define the norm

$$
\|\varphi\|_{q ; \rho} \equiv\left(\int_{B(x, \rho)}|\varphi|^{q} d V_{0}\right)^{1 / q}
$$

\|\|$_{q}$ will mean the $L^{q}$-norm with respect to $d V_{0}$ (unless indicated otherwise).

We will also use the following well-known result from elliptic theory: Given $x \in M, \rho>0, q>n$, then

$$
\sup _{B(x, \rho / 2)}|\varphi| \leq C\left(\|\Delta \varphi\|_{q ; \rho}+\|\varphi\|_{q ; \rho}\right) .
$$

\section{$\S 2$. Analysis of the Blow Up}

In this section we show that if the sequence $\left\{u_{k}\right\}$ is not bounded, then it blows up at finitely many points, and off these blow-up points we may extract a subsequence which converges uniformly on compacta. 
Proposition 2.1. Given a sequence of smooth positive functions $\left\{u_{k}\right\}$ which satisfy

$$
\begin{aligned}
& L_{g_{0}} u_{k}=-\alpha_{n} R_{k} u_{k}^{N-1} \\
& \int\left|R_{k}\right|^{p} u_{k}^{N} d V_{0} \leq \beta^{p} \quad(p>n / 2) \\
& \int u_{k}^{N} d V_{0} \leq V_{0}
\end{aligned}
$$

then either (i) there is a constant $C=C\left(V_{0}, \beta\right)>0$ independent of $k$ such that

$$
\max _{M} u_{k} \leq C
$$

or (ii) there is a finite set of points $\Sigma=\left\{x_{1}, x_{2}, \ldots, x_{\nu}\right\}$ with $\nu=\nu\left(V_{0}, \beta\right)$ such that given a compact set $K \subset \tilde{M} \equiv M-\Sigma$, there is a constant $C\left(K, V_{0}, \beta\right)$ which is independent of $k$ such that

$$
\max _{K} u_{k} \leq C\left(K, V_{0}, \beta\right)
$$

(hence a further subsequence converges in $C^{\alpha}(K)$ for some $\alpha>0$ by $(2.1)$ and $(2.2))$.

Before giving the details of the Proof of Proposition 2.1, it will be helpful to record the following regularity result for weak solutions of certain elliptic equations, of which (2.1) is an example. To simplify the exposition, we postpone its proof until the end of the section.

Lemma 2.2. Suppose $w \in W^{1,2}(M)$ is a non-negative, weak solution of

$$
L_{g_{0}} w=f w^{r}
$$


where

$$
\int|f|^{p} d V_{0} \leq \beta^{p} \quad(p>n / 2)
$$

and $1 \leq r \leq r_{0}=(N-1)-\frac{1}{p} N$. Then given $x \in M, \rho>0, q>N$ we have

$$
\sup _{B(x, \rho / 3)} w \leq C\left(\rho, \beta, p, q,\|w\|_{q ; \rho}\right)
$$

Note that $u_{k}$ satisfies an equation of type (2.4) with $f=-\alpha_{n} R_{k} u_{k}^{\frac{1}{p} N}, r=r_{0}$.

Proof (of Proposition 2.1). Given $x \in M$, following [CY2] we define

$$
\begin{aligned}
m(x) & =\text { mass of } x \\
& =\lim _{r \rightarrow 0} \limsup _{k \rightarrow \infty} \int_{B(x, \rho)} u_{k}^{N} d V_{0},
\end{aligned}
$$

and $\Sigma=\{x \in M: m(x)>0\}$. The term mass comes from the interpretation of the function $u_{k}^{N}$ as a density distribution. A point $x \in M$ will have large mass if the sequence $\left\{u_{k}\right\}$ "concentrates" at $x$. However, if the mass of $x$ is small enough, we will see that $\left\{u_{k}\right\}$ is in fact bounded in a small neighborhood of $x$.

LEMMA 2.3. Given $x \in M$, either $m(x)=0$ or $m(x) \geq \mu \equiv\left(8 A_{0} \alpha_{n} \beta\right)^{-\frac{n_{P}}{2 P-n}}$ (see (1.0) for the definition of $A_{0}$ ).

Proof. Suppose $x \in M$ such that $0 \leq m(x)<\mu$. Fix $r>0$ small enough and an integer $J$ large enough so that $k \geq J$ implies

$$
\int_{B(x, r)} u_{k}^{N} d V_{0} \leq \mu .
$$


Let $\eta$ be a smooth cut-off function, $0 \leq \eta \leq 1$, with $\eta \equiv 1$ on $B(x, r / 2)$ and $\eta \equiv 0$ on $B^{c}(x, r)$, and $|\nabla \eta| \leq C / r$. Multiplying (2.1) by $\eta^{2} u_{k}^{1+2 \varepsilon}$ with $\varepsilon$ chosen so that $2+2 \varepsilon=N$, and integrating by parts yields

$$
\begin{aligned}
(1+2 \varepsilon) \int u_{k}^{2 \varepsilon} \eta^{2}\left|\nabla u_{k}\right|^{2} d V_{0}= & -2 \int u^{1+2 \varepsilon} \eta \nabla u_{k} \nabla \eta d V_{0} \\
& -\alpha_{n} \int R_{0} u_{k}^{2+2 \varepsilon} \eta^{2} d V_{0} \\
& +\alpha_{n} \int R_{k} u_{k}^{N+2 \varepsilon} \eta^{2} d V_{0} \\
\Rightarrow \quad \int\left|\nabla\left(\eta u_{k}^{1+\varepsilon}\right)\right|^{2} d V_{0} & \leq 4 \alpha_{n} \int\left|R_{k}\right| u_{k}^{N+2 \varepsilon} \eta^{2} d V_{0} \\
& +\frac{C}{r^{2}} \int u_{k}^{2+2 \varepsilon} d V_{0} .
\end{aligned}
$$

This implies, by the Sobolev inequality (1.1) and the choice of $\varepsilon$

$$
\begin{aligned}
\left\|\eta u_{k}^{1+\varepsilon}\right\|_{N}^{2} \leq & 4 A_{0} \alpha_{n} \int\left|R_{k}\right| u_{k}^{N-2} u_{k}^{2+2 \varepsilon} \eta^{2} d V_{0} \\
& +\frac{C^{\prime}\left(V_{0}\right)}{r^{2}} \\
& \leq 4 A_{0} \alpha_{n}\left(\int_{\text {supp } \eta}\left|R_{k}\right|^{\frac{n}{2}} u_{k}^{N} d V_{0}\right)^{\frac{2}{n}}\left\|\eta u_{k}^{1+\varepsilon}\right\|_{N}^{2} \\
& +\frac{C^{\prime}}{r^{2}} \\
& \leq 4 A_{0} \alpha_{n} \beta\left\|u_{k}\right\|_{N ; \rho}^{N\left(\frac{2 p-n}{n p}\right)}\left\|\eta u_{k}\right\|_{N}^{2} \\
& \leq 4 A_{0} \alpha_{n} \beta \mu^{\frac{2 p-n}{n p}}\left\|\eta u_{k}^{1+\varepsilon}\right\|_{N}^{2}+\frac{C^{\prime}}{r^{2}} \\
& \leq \frac{1}{2}\left\|\eta u_{k}^{1+\varepsilon}\right\|_{N}^{2}+\frac{C^{\prime}}{r^{2}} \\
\left\|\eta u_{k}^{1+\varepsilon}\right\|_{N}^{2} & \leq \frac{C^{\prime}}{r^{2}} .
\end{aligned}
$$


It follows easily from (2.8) that $m(x)=0$. For, given $0<\rho<r / 2$ and any $k \geq J$,

$$
\begin{aligned}
\int_{B(x, \rho)} u_{k}^{N} d V_{0} & \leq\left\|u_{k}^{1+\varepsilon}\right\|_{N ; r / 2}^{N /(1+\varepsilon)}\left(\int_{B(x, \rho)} d V_{0}\right)^{\frac{\varepsilon}{1+\varepsilon}} \\
& \leq\left(\frac{C^{\prime}}{r^{2}}\right)^{\frac{N}{2(1+\varepsilon}} C_{n} \rho^{\frac{n e}{1+\varepsilon}}
\end{aligned}
$$

and taking the appropriate limits shows $m(x)=0$. This completes the proof of the lemma.

Given $x \in M$ with $m(x)=0$, then (2.8) holds for some $r=r(x)$ and all $k \geq J(x)$. Hence, by (2.6) we have

$$
\max _{B(x, r / 6)} u_{k} \leq C\left(V_{0}, \beta, r\right)
$$

for all $k \geq J(x)$. In particular, if $\Sigma=\phi$ then we may cover $M$ with finitely many balls (by compactness), on each of which the estimate (2.9) holds, and it follows that $\left\{u_{k}\right\}$ in fact has a uniform upper bound.

To complete the proof of the proposition, suppose $\Sigma$ does not have finitely many members, and choose a sequence $\left\{x_{i}\right\}$ of distinct elements from $\Sigma$.

Now for $x_{1}$, choose a subsequence of $\left\{u_{k}\right\}$, call it $\left\{u_{k_{1}}\right\}$ such that

$$
\lim _{r \rightarrow 0} \lim _{k_{1} \rightarrow \infty} \int_{B\left(x_{1}, r\right)} u_{k_{1}}^{N} d V_{0}=m\left(x_{1}\right) \text {. }
$$

Now choose a subsequence $\left\{u_{k_{2}}\right\}$ of $\left\{u_{k_{1}}\right\}$ such that

$$
\lim _{r \rightarrow 0} \lim _{k_{2} \rightarrow \infty} \int_{B\left(x_{2}, r\right)} u_{k_{2}}^{N} d V_{0}=m\left(x_{2}\right)
$$


and so on, and take a diagonal subsequence which we again denote by $\left\{u_{k}\right\}$. Now $m\left(x_{i}\right) \geq \mu$.

Let $\ell$ be a large enough integer so that

$$
\ell(\mu / 2) \geq 2 V_{0}
$$

Let $r>0$ be small enough and $J$ large enough so that for $1 \leq i \leq \ell, k \geq J$,

$$
\int_{B\left(x_{i}, r\right)} u_{k}^{N} d V_{0} \geq \mu / 2
$$

and we also assume that $r$ is small enough so that $B\left(x_{i}, r\right) \cap B\left(x_{j}, r\right)=\phi$ if $i \neq j$. Then

$$
V_{0} \geq \sum_{i=1}^{\ell} \int_{B\left(\boldsymbol{x}_{i}, \boldsymbol{r}\right)} u_{k}^{N} d V_{0} \geq \ell(\mu / 2) \geq 2 V_{0},
$$

a contradiction. Hence $\Sigma$ consists of finitely many points $\left\{x_{1}, \ldots, x_{\nu}\right\}$, and we have the estimate $\nu \lesssim \mu^{-1} V_{0}$. If $K$ is a compact set, $K \subset \tilde{M}$, then we may cover $K$ with finitely many balls, on each of which the estimate (2.9) holds, and conclude that for $k \geq J(K)$,

$$
\max _{K} u_{k} \leq C\left(V_{0}, \beta, K\right)
$$

This finishes the proof of the proposition.

By Proposition 2.1, a subsequence of $\left\{u_{k}\right\}$ has a limit

$$
w=\lim _{k} u_{k}
$$


which is defined on $\tilde{M}$ (or on $M$ in case $\left\{u_{k}\right\}$ is bounded) and is Hölder continuous on compact $K \subset \tilde{M}$. If $\Sigma \neq \phi$, there remains the possibility that $w$ has a singularity at some $x_{i} \in \Sigma$, but in fact this is not the case, as the following proposition shows.

Proposition 2.4. $w \leq C\left(V_{0}, \beta\right)$.

Proof. Let us assume that $\Sigma \neq \phi$; otherwise the result is obvious. Since $w$ is defined on $\tilde{M}$, we begin the Proof of Proposition 2.4 by showing that $w$ weakly satisfies a certain elliptic equation on $\tilde{M}$. To this end, note that the bound

$$
\int\left|R_{k} u_{k}^{\frac{1}{p} N}\right|^{p} d V_{0} \leq \beta^{p}
$$

along with the fact that $p>1$ implies that $\left\{-\alpha_{n} R_{k} u_{k}^{\frac{1}{p} N}\right\}$ has a subsequence which converges weakly in $L^{p}$ to $f \in L^{p}$; i.e., given $\psi \in L^{p^{\prime}}, \frac{1}{p}+\frac{1}{p^{\prime}}=1$, then

$$
\lim _{k}-\alpha_{n} \int R_{k} u_{k}^{\frac{1}{p} N} \psi d V_{0}=\int f \psi d V_{0}
$$

and $\int|f|^{p} d V_{0} \leq \beta^{p}$

LEMMA 2.5. $w$ satisfies the elliptic equation

$$
L_{g_{0}} w=f w^{r}
$$

weakly on $\tilde{M}$, where $\frac{1}{p} N+r=N-1$. 
Proof. Let $\varphi \in C_{0}^{\infty}(\tilde{M})$. Let $\left\{u_{k}\right\}$ denote a subsequence which converges uniformly to $w$ on the support of $\varphi$. Then

$$
\begin{aligned}
\int\left(L_{g_{0}} \varphi\right) w d V_{0} & =\int\left(L_{g_{0}} \varphi\right)\left(w-u_{k}\right) d V_{0}+\int\left(L_{g_{0}} \varphi\right) u_{k} d V_{0} \\
& =I_{1}+\int \varphi\left(L_{g_{0}} u_{k}\right) d V_{0} \\
& =I_{1}-\alpha_{n} \int R_{k} u_{k}^{N-1} \varphi d V_{0} \\
& =I_{1}-\alpha_{n} \int R_{k} u_{k}^{\frac{1}{p} N}\left(u_{k}^{r}-w^{r}\right) \varphi d V_{0} \\
& \quad-\alpha_{n} \int R_{k} u_{k}^{\frac{1}{p} N} w^{r} \varphi d V_{0} \\
& =I_{1}+I_{2}+I_{3} .
\end{aligned}
$$

Since $u_{k} \rightarrow w$ uniformly on the support of $\varphi$, we see that $I_{1}, I_{2} \rightarrow 0$ as $k \rightarrow \infty$. Also, $\varphi w^{r}$ is bounded (hence in $L^{p^{\prime}}$ ) so by weak convergence,

$$
-\alpha_{n} \int R_{k} u_{k}^{\frac{1}{p} N} w^{r} \varphi d V_{0} \rightarrow \int f w^{r} \varphi d V_{0} \quad k \rightarrow \infty
$$

which proves the lemma.

The following weak removable singularities result can be found in [LP]. We will use it to show that (2.10) holds weakly on all of $M$.

Lemma 2.6 (see [LP]). Let $U$ be an open set in $M$ and $P \in U$. Suppose $w$ is a weak solution of $(\Delta+h) w=0$ on $U-\{P\}$, with $h \in L^{n / 2}(U)$ and $w \in L^{q}(U)$ for some $q>\frac{n}{n-2}$. Then w satisfies $(\Delta+h) w=0$ weakly on all of $U$.

Lemma 2.7. Equation (2.10) holds weakly on $M$. 
Proof (of Lemma 2.7). We apply Lemma 2.6 with

$$
h=-\alpha_{n} R_{0}-f w^{r-1} .
$$

We need to verify that $h \in L^{n / 2}(M)$ (by the definition of $w$ it is clear that $\left.w \in L^{n / n-2}(M)\right)$. But by Hölder and the definition of $r$,

$$
\begin{aligned}
\int\left|f w^{r-1}\right|^{\frac{n}{2}} & \leq\|f\|_{p}^{n / 2}\|w\|_{N}^{\frac{n}{2}(r-1)} \\
& \leq C\left(\beta, V_{0}\right)
\end{aligned}
$$

The boundedness of $w$ now follows from the fact that $w$ satisfies (2.10) weakly on $M$ and some standard elliptic estimates. A good reference for this argument would be [T], but for the sake of completeness we give the details here.

Let $x_{i} \in \Sigma, \rho>0$ a small number to be chosen later. Let $\eta$ be a smooth cut-off function, $0 \leq \eta \leq 1$ with $\eta \equiv 1$ on $B\left(x_{i}, \rho / 2\right)$ and $\eta \equiv 0$ on $B^{c}\left(x_{i}, \rho\right)$. Let $\alpha, k, F, G$ be as in the Proof of Lemma 2.2. Then by (2.13) we have (with $\xi=\eta F(w))$

$$
\begin{aligned}
\|\xi\|_{N}^{2} \leq 4 \alpha A_{0} & \int|f| w^{r-1} \xi^{2} d V_{0} \\
& +C \frac{\alpha^{2}}{\rho^{2}} \int(F(w))^{2} d V_{0}
\end{aligned}
$$

Now

$$
\int|f| w^{r-1} \xi^{2} d V_{0} \leq\|f\|_{\rho}\|w\|_{N ; \rho}^{r-1}\|\xi\|_{N}^{2}
$$


Let $\alpha=N / 2$. Since $w \in L^{N}(M)$, we can fix $\rho$ small enough so that

$$
\|w\|_{N ; \rho}^{r-1} \leq\left(4 N A_{0} \beta\right)^{-1}
$$

Then (2.11) becomes

$$
\|\xi\|_{N}^{2} \leq \frac{1}{2}\|\xi\|_{N}^{2}+C(\rho) \int(F(w))^{2} d V_{0}
$$

Letting $k \rightarrow \infty$ we have

$$
\left\|w^{N / 2}\right\|_{N ; \rho / 2} \leq C\left(V_{0}, \beta\right)
$$

Since $N^{2} / 2>N$, we may apply Lemma 2.2 and conclude

$$
\sup _{B\left(x_{i}, \rho / 6\right)} w \leq C\left(V_{0}, \beta\right)
$$

and this completes the Proof of Proposition 2.4.

We now give the Proof of Lemma 2.2. Let $\alpha, k \geq 1$ and define $F, G \in C^{1}[0, \infty)$ by

$$
\begin{gathered}
F(t)=\left\{\begin{array}{lll}
t^{\alpha} & \text { if } & t \in[0, k], \\
k^{\alpha}+\alpha k^{\alpha-1}(t-k) & \text { if } & t>k ;
\end{array}\right. \\
G(t)=\left\{\begin{array}{lll}
t^{2 \alpha-1} & \text { if } t \in[0, k], \\
k^{2 \alpha-1}+(2 \alpha-1) k^{2 \alpha-2}(t-k) & \text { if } t>k .
\end{array}\right.
\end{gathered}
$$

Then $F(w), G(w) \in W^{1,2}(M)$.

Let $\eta$ be a smooth cut-off function supported in some geodesic ball $B_{\rho} \equiv$ $B(x, \rho)$ whose properties we will specify later. Let $v=\eta^{2} G(w)$; then as $w$ 
satisfies (2.4) weakly we have

$$
\begin{aligned}
-\int \nabla w \nabla v d V_{0} & -\alpha_{n} \int R_{0} w v=\int f w^{r} v d V_{0} \\
\Rightarrow \quad \int \eta^{2} G^{\prime}(w)|\nabla w|^{2} d V_{0} & +2 \int \eta G(w) \nabla w \nabla \eta d V_{0} \\
& =-\alpha_{n} \int R_{0} w v d V_{0}+\int f w^{r} v d V_{0}
\end{aligned}
$$

Now

$$
\begin{aligned}
2 \eta G(w)|\nabla w \nabla \eta| \leq \frac{1}{2} \eta^{2} & \frac{G(w)}{w}|\nabla w|^{2} \\
& +2|\nabla \eta|^{2} w G(w)
\end{aligned}
$$

and it is easily verified that

$$
G(w) \leq w G^{\prime}(w), w G(w) \leq(F(w))^{2},\left(F^{\prime}(w)\right)^{2} \leq \alpha G^{\prime}(w)
$$

Hence

$$
\begin{aligned}
\frac{1}{2 \alpha} \int \eta^{2}\left(F^{\prime}(w)\right)^{2}|\nabla w|^{2} d V_{0} & \leq \frac{1}{2} \int|\nabla \eta|^{2}(F(w))^{2} d V_{0} \\
& +\alpha_{n} \int\left|R_{0}\right|(F(w))^{2} \eta^{2} d V_{0} \\
& +\int|f| w^{r-1}(F(w))^{2} \eta^{2} d V_{0}
\end{aligned}
$$

Since $|\nabla(\eta F(w))|^{2} \leq 2 \eta^{2}\left(F^{\prime}(w)\right)^{2}|\nabla w|^{2}+2(F(w))^{2}|\nabla \eta|^{2}$, letting $\xi=\eta F(w)$ we have

$$
\begin{aligned}
\int|\nabla \xi|^{2} d V_{0} \leq & 2 \alpha(1+\alpha) \int(F(w))^{2}|\nabla \eta|^{2} d V_{0} \\
& +4 \alpha \alpha_{n} \int\left|R_{0}\right| \xi^{2} d V_{0} \\
& +4 \alpha \int|f| w^{r-1} \xi^{2} d V_{0}
\end{aligned}
$$


Using the Sobolev inequality (1.1) we see that

$$
\begin{aligned}
\|\xi\|_{N}^{2} \leq 4 \alpha A_{0} & \int|f| w^{r-1} \xi^{2} d V_{0} \\
& +C \alpha^{2} \int\left(\eta^{2}+|\nabla \eta|^{2}\right)(F(w))^{2} d V_{0}
\end{aligned}
$$

Our intention is to iterate (2.13) in order to show that $w \in L^{s}\left(B_{\rho / 2}\right)$ for some $s>N$. For example, suppose we can show that $\|w\|_{s ; \rho / 2} \leq \gamma<\infty$ with

$$
s \geq s_{0} \equiv \frac{p t}{p-t} r
$$

where $p>t>\frac{n}{2}$. Then by Hölder,

$$
\begin{aligned}
\left\|L_{g_{0}} w\right\|_{t ; p / 2} & \leq\|f\|_{p}\|w\|_{s ; p / 2}^{r} \\
& \leq \beta \gamma^{r} .
\end{aligned}
$$

This implies, by elliptic regularity and the Sobolev imbedding that

$$
\sup _{B_{\rho / 4}} w \leq C(\beta, \gamma, \rho)
$$

Hence, to prove (2.6) it suffices to show that $\|w\|_{s ; \rho / 2} \leq C\left(\beta, \rho,\|w\|_{q ; \rho}\right)$. We now proceed to do this.

To set up the iteration, we analyze the first integral on the RHS of (2.13):

$$
\int|f| w^{r-1} \xi^{2} d V_{0} \leq\|f\|_{\rho}\|w\|_{q ; \rho}^{r-1}\|\xi\|_{\sigma}^{2}
$$


where $\sigma=2 p q /[(p-1) q-p(r-1)]$. It is easily verified that the requirements $p>n / 2, q>N, r \leq r_{0}$ imply $\sigma<N$. Hence (2.13) implies

$$
\begin{aligned}
\|\xi\|_{N}^{2} \leq 4 \alpha A_{0} \beta\|w\|_{q ; \rho}^{r-1}\|\xi\|_{\sigma}^{2} \\
+C \alpha^{2} \int\left(\eta^{2}+|\nabla \eta|^{2}\right)(F(w))^{2} d V_{0} \\
\Rightarrow \quad\|\xi\|_{N} \leq \Lambda\left(\beta, \alpha, \rho,\|w\|_{q ; \rho}\right)\left(\int_{\text {supp } \eta}(F(w))^{\sigma} d V_{0}\right)^{1 / \sigma}
\end{aligned}
$$

We now specify our cut-off function $\eta$ in order to iterate (2.16).

Let $J$ be a fixed integer satisfying $N(N / \sigma)^{J}>s_{0}$, where $s_{0}$ is defined in (2.14). For $1 \leq j \leq J$, let

$$
\begin{aligned}
& \rho_{j}=\rho[1-j / 2 J], \\
& \rho_{0}=\rho .
\end{aligned}
$$

Then $\rho=\rho_{0}>\rho_{1}>\cdots>\rho_{J-1}>\rho_{J}=\rho / 2$. Let $\eta_{j}$ be a smooth cut-off function, $0 \leq \eta_{j} \leq 1, \eta_{j} \equiv 1$ on $B_{\rho_{j}}$ and $\eta_{j} \equiv 0$ on $B_{\rho_{j-1}}^{c}$. Then

$$
\operatorname{supp} \eta_{j} \subset\left\{x: \eta_{j-1}=1\right\}
$$

For $1 \leq j \leq J$, let $\alpha_{j}=(N / \sigma)^{j}$; then letting $k \rightarrow \infty$ (recall the definitions of $F$ and $\xi)$ in (2.16) we have

$$
\begin{aligned}
\left\|w^{\alpha_{J}} \eta_{J}\right\|_{N} & \leq \Lambda\left(\int_{\text {supp } \eta_{J}} w^{\sigma \alpha_{J}} d V_{0}\right)^{1 / \sigma} \\
& \leq \Lambda\left(\int\left(w^{\alpha_{J}(\sigma / N)} \eta_{J-1}\right)^{N} d V_{0}\right)^{1 / \sigma} \\
& \leq \Lambda\left\|w^{\alpha_{J-1}} \eta_{J-1}\right\|_{N}^{(N / \sigma)} .
\end{aligned}
$$


Repeating this argument we arrive at

$$
\begin{aligned}
\left\|w^{\alpha_{J}} \eta_{J}\right\|_{N} & \leq \Lambda^{J-1}\left\|w^{\alpha_{1}} \eta_{1}\right\|_{N}^{(N / \sigma)^{J-1}} \\
& \leq \Lambda^{J}\|w\|_{N}^{(N / \sigma)^{J}}
\end{aligned}
$$

By the definition of $\alpha_{J}$ and $\eta_{J}$ this implies

$$
\|w\|_{s_{0} ; \rho / 2} \leq C\left(\beta, \rho,\|w\|_{q ; \rho}\right)
$$

so by our earlier comments (in particular (2.15)) this completes the Proof of the Lemma.

\section{§3. FINDING AN UPPER BOUND}

In the previous section we saw that the sequence of conformal factors $\left\{u_{k}\right\}$ has a Hölder continuous limit $w$. In practice, non-compactness results in the metrics degenerating in such a way that $w \equiv 0$. But if $\left\{u_{k}\right\}$ has a positive uniform lower bound, then in fact it has a uniform upper bound. To be more precise,

Proposition 3.1. Suppose there is a constant $c_{0}>0$ such that $u_{k} \geq c_{0}$ for all $k$. Then $\Sigma=\phi$ and therefore by Proposition 2.1, $\left\{u_{k}\right\}$ satisfies the conclusions of the Theorem $(0.3),(0.4)$.

The Proof of Proposition 2.1 will be divided into several steps, some involving lengthy calculations, so we begin by attempting to motivate the proof.

Our first observation is the fact that the function $1 / u_{k}$ (for which we have upper bounds) is natural to consider in view of the "conformal covariance" of $L$. 
For,

$$
L_{g_{k}}\left(1 / u_{k}\right)=u_{k}^{-N+1} L_{g_{0}}(1)=-\alpha_{n} R_{0} u_{k}^{-N+1} .
$$

Letting $\varphi_{k}=1 / u_{k}$ we rewrite this as

$$
\Delta_{g_{k}} \varphi_{k}=\alpha_{n} R_{k} \varphi_{k}-\alpha_{n} R_{0} \varphi_{k}^{N-1}
$$

and conclude that $1 / u_{k}$ satisfies an elliptic equation in the metric $g_{k}$. As a consequence, one might anticipate that upper bounds on $\varphi_{k}$ would imply even stronger regularity properties, provided we have an elliptic regularity result for the Laplacian $\Delta_{g_{k}}$. It turns out that using the Bochner identity and the $L^{p}$ curvature bounds for $g_{k}$ one can prove such an estimate (Proposition 3.3). The improved regularity thus achieved is given a precise statement in

Proposition 3.2. If $n \geq 4$ then there are constants $s_{0}=[p(n+2)-2 n] / p(n-$ 2) $>0, r_{0}=n p /(n-p)>n, C_{3}=C_{3}\left(c_{0}, V_{0}, \beta\right)$ such that

$$
\int\left|\nabla_{0}\left(u_{k}^{-s_{0}}\right)\right|^{r_{0}} d V_{0} \leq C_{3}
$$

As a consequence, there is a constant $C_{4}=C_{4}\left(C_{0}, C_{3}\right)$ such that

$$
\left\|u_{k}^{-s_{0}}\right\|_{C^{\alpha}(M)} \leq C_{4},
$$

where $\alpha=(2 p-n) / p>0$.

If $n=3$, then the same conclusions hold with $s_{0}=2, r_{0}=6, \alpha=1 / 2 ;$ but $C_{2}$ and $C_{3}$ will also depend on $\tilde{\beta}$. 
Let us see how to conclude Proposition 3.1 assuming Proposition 3.2. Suppose $\Sigma \neq \phi$ and choose $x_{1} \in \Sigma$. Consider a small geodesic ball $B=B_{\rho}\left(x_{1}\right)$ with

$$
\rho=\left[2^{-s_{0}-\alpha-1}\|w\|_{\infty}^{-s_{0}} C_{3}^{-1}\right]^{1 / \alpha} .
$$

The purpose of this choice of $\rho$ will become apparent later, but for now think of $\rho$ as a fixed positive number. Fix $P \in \partial B$ and let $Q \in B$ be arbitrary. Then $d(P, Q) \leq 2 \rho$, and for all sufficiently large $k$ (say $k \geq J_{0}$ ) we have $u_{k}(P) \leq$ $2\|w\|_{\infty}$. By (3.3), for $k \geq J_{0}$,

$$
\begin{aligned}
&\left|u_{k}^{-s_{0}}(P)-u_{k}^{-s_{0}}(Q)\right| \leq C_{3} d(P, Q)^{\alpha} \\
& \Rightarrow \quad u_{k}^{-s_{0}}(Q) \geq u_{k}^{-s_{0}}(P)-C_{3} d(P, Q)^{\alpha} \\
& \geq 2^{-s_{0}}\|w\|_{\infty}^{-s_{0}}-C_{3} 2^{\alpha} \rho^{\alpha} \\
& \geq 2^{-s_{0}}\|w\|_{\infty}^{-s_{0}}-C_{3} 2^{\alpha}\left[2^{-s_{0}-\alpha-1}\|w\|_{\infty}^{-s_{0}} C_{3}^{-1}\right] \\
& \geq 2^{-s_{0}-1}\|w\|_{\infty}^{-s_{0}} \\
& \Rightarrow \quad u_{k}(Q) \leq 2^{1+1 / s_{0}}\|w\|_{\infty}
\end{aligned}
$$

and this estimate holds for all $Q \in B, k \geq J_{0}$. But this implies, of course, that $m\left(x_{1}\right)=0$, contradicting $x_{1} \in \Sigma$. Hence $\Sigma=\phi$.

We have shown that Proposition 3.1 follows from Proposition 3.2. In order to prove Proposition 3.2, we begin by stating the elliptic regularity result mentioned earlier. 
Proposition 3.3. Let $(M, h)$ be a compact $n$-dimensional manifold, $n \geq 3$. Let $A_{h}$ denote the Sobolev constant of $(M, h)$; i.e., for all $f \in W^{1,2}(M, h)$,

$$
\left(\int|f|^{N} d V_{h}\right)^{2 / N} \leq A_{h}\left\{\int\left|\nabla_{h} f\right|^{2} d V_{h}+\int f^{2} d V_{h}\right\}
$$

Then for all $\varphi \in C^{\infty}(M), t \geq 1, p>n / 2$,

$$
\begin{array}{r}
\left(\int\left|\nabla_{h} \varphi\right|^{N t} d V_{h}\right)^{2 / N} \leq B_{1} \int\left|\nabla_{h} \varphi\right|^{2 t-2}\left(\Delta_{h} \varphi\right)^{2} d V_{h} \\
+B_{2} \int\left|\nabla_{h} \varphi\right|^{2 t} d V_{h}
\end{array}
$$

where

$$
\begin{aligned}
& B_{1}=2 A_{h} t^{2}(1+t), \\
& B_{2}=2 A_{h}\left\{t^{2}\left[2 A_{h} t^{2}\left\|R c_{h}\right\|_{p}^{2 p / n}\right]^{n /(2 p-n)}+1\right\}
\end{aligned}
$$

and

$$
\left\|R c_{h}\right\|_{p}=\left(\int\left|R c_{h}\right|^{p} d V_{h}\right)^{1 / p}
$$

Proving (3.3) will involve some elementary but nevertheless tedious calculations, so let us first show how to conclude Proposition 3.2 from Proposition (3.3). The idea behind the proof is rather simple. Observe that

$$
\begin{aligned}
\int\left|\nabla_{g_{k}}\left(u_{k}^{-1}\right)\right|^{2} d V_{g_{k}} & =\int\left|\nabla_{0} u_{k}\right|^{2} u_{k}^{-2} d V_{0} \\
& \leq C\left(c_{0}, V_{0}, \beta\right) .
\end{aligned}
$$


Using this initial bound, we then iterate inequality (3.5) with $h=g_{k}$ and $\varphi=$ $1 / u_{k}$ in order to arrive at the estimate (3.2). In order to set up the iteration, however, we need to do some preliminary work.

Since we want our estimates to be independent of $k$, we need to show that the Sobolev constants $A_{g_{k}}$ have a uniform upper bound. Of course the lower bound on $u_{k}$ implies a lower bound on the injectivity radius of $g_{k}$, and this, combined with the upper bound on volume (0.1), gives control of the Sobolev constant (see, for example [Cr]); but there is a more natural way to see this in the conformal setting, given our curvature bounds.

Let $\varphi=u_{k} f$; then by conformal invariance of the Dirichlet energy we have $E_{g_{k}}(f)=E_{g_{0}}(\varphi)$, so by the Sobolev inequality (1.1)

$$
\begin{aligned}
\left(\int|f|^{N} d V_{g_{k}}\right)^{2 / N} & =\left(\int|\varphi|^{N} d V_{0}\right)^{2 / N} \\
& \leq A_{0}\left[\int\left|\nabla_{0} \varphi\right|^{2} d V_{0}+\int \varphi^{2} d V_{0}\right] \\
& \leq A_{0}\left[E_{g_{0}}(\varphi)+\int\left(1-\alpha_{n} R_{0}\right) \varphi^{2} d V_{0}\right] \\
& \leq A_{0}\left[E_{g_{k}}(f)+\int\left(1-\alpha_{n} R_{0}\right) f^{2} u_{k}^{-N+2} d V_{0}\right] \\
& \leq A_{0}\left[\int\left|\nabla_{g_{k}} f\right|^{2} d V_{g_{k}}+\alpha_{n} \int R_{k} f^{2} d V_{g_{k}}\right. \\
& \left.\quad+C\left(C_{0}\right) \int f^{2} d V_{g_{k}}\right] .
\end{aligned}
$$


Let $b>>0$ and define $E_{b}=\left\{x \in M: \mid R_{k}(x / \mid \geq b\}\right.$. Then

$$
\begin{aligned}
\int R_{k} f^{2} d V_{g_{k}} & =\int_{E_{b}^{c}} R_{k} f^{2} d V_{g_{k}}+\int_{E_{b}} R_{k} f^{2} d V_{g_{k}} \\
& \leq b \int f^{2} d V_{g_{k}}+\left(\int_{E_{b}}\left|R_{k}\right|^{\frac{n}{2}} d V_{g_{k}}\right)^{\frac{2}{n}}\left(\int|f|^{N} d V_{g_{k}}\right)^{2 / N} .
\end{aligned}
$$

Since

$$
\beta^{p} \geq \int_{E_{b}}\left|R_{k}\right|^{p} d V_{g_{k}} \geq b^{p-\frac{n}{2}} \int_{E_{b}}\left|R_{k}\right|^{n / 2} d V_{g_{k}},
$$

we have

$$
\left(\int_{E_{b}}\left|R_{k}\right|^{\frac{n}{2}} d V_{g_{k}}\right)^{2 / n} \leq b^{-\frac{2}{n}\left(p-\frac{n}{2}\right)} \beta^{2 p / n} .
$$

Substituting this into (3.8), and (3.8) into (3.7) we find

$$
\begin{aligned}
\left(\int|f|^{N} d V_{g_{k}}\right)^{2 / N} \leq \alpha_{n} A_{0} b^{-\frac{2}{n}\left(p-\frac{n}{2}\right)} \beta^{2 p / n}\left(\int|f|^{N} d V_{g_{k}}\right)^{2 / N} & \\
& +A_{0} \int\left|\nabla_{g_{k}} f\right|^{2} d V_{g_{k}}+C\left(b, c_{0}\right) \int f^{2} d V_{g_{k}}
\end{aligned}
$$

Choosing $b$ large enough (depending on $\beta$ ) we can absorb the first term on the RHS of the above inequality into the LHS, giving us an upper bound for $A_{g_{k}}$ depending on $c_{0}$ and $\beta$ which we will henceforth denote by $A_{0}$.

If we take $h=g_{k}, \varphi=1 / u_{k}$ in (3.5), then by (3.1), we have (after dropping subscripts to simplify notation)

$$
\begin{gathered}
\left(\int|\nabla \varphi|^{N t} d V\right)^{2 / N} \leq B_{1} \int|\nabla \varphi|^{2 t-2}\left(\alpha_{n} R \varphi-\alpha_{n} R_{0} \varphi^{N-1}\right)^{2} d V \\
+B_{2} \int|\nabla \varphi|^{2 t} d V
\end{gathered}
$$


Considering the first term on the RHS of (3.9) we have

$$
\begin{aligned}
& \int|\nabla \varphi|^{2 i-2}\left(\alpha_{n} R \varphi-\alpha_{n} R_{0} \varphi^{N-1}\right)^{2} d V \\
& \quad \leq 2 c_{0}^{2} \alpha_{0}^{2} \int|\nabla \varphi|^{2 t-2} R^{2} d V+2 \alpha_{n}^{2}\left\|R_{0}\right\|_{\infty}^{2} c_{0}^{2(N-1)} \int|\nabla \varphi|^{2 t-2} d V
\end{aligned}
$$

Now for $\delta>0$,

$$
\begin{aligned}
\int|\nabla \varphi|^{2 t-2} R^{2} d V & \leq\left(\int|\nabla \varphi|^{N t} d V\right)^{\frac{2(t-1)}{N t}}\left(\int|R|^{\frac{2 N t}{N t+2(1-t)}} d V\right)^{\frac{N t+2(1-t)}{N t}} \\
& \leq \frac{t-1}{t} \delta\left(\int|\nabla \varphi|^{N t} d V\right)^{2 / N}+\frac{1}{t} \delta^{1-t}\|R\|_{\frac{2 N t}{N t+2(1-t)}}^{2}
\end{aligned}
$$

where here, and in what follows, \|\|$_{s}$ denotes the $L^{s}$-norm in the $g_{k}$ metric. If we take

$$
\delta=\left(4 c_{0}^{2} \alpha_{n}^{2} B_{1}\right)^{-1}
$$

and substitute (3.11) into (3.10), and (3.10) into (3.9) we find

$$
\begin{aligned}
&\|\nabla \varphi\|_{N t}^{2 t} \leq \frac{1}{2}\|\nabla \varphi\|_{N t}^{2 t}+C\left(B_{1}, c_{0}\right)\|R\|_{\frac{2 N t}{N t+2(1-t)}}^{2} \\
&+C\left(B_{2}, c_{0}\right)\|\nabla \varphi\|_{2 t}^{2 t} \\
& \Rightarrow \quad\|\nabla \varphi\|_{N t} \leq C\left(B_{1}, c_{0}\right)\|R\|_{\frac{2 N t}{N t+2(1-t)}}^{1 / t}+C\left(B_{2}, c_{0}\right)\|\nabla \varphi\|_{2 t} .
\end{aligned}
$$

Inequality (3.12) makes it clear why we need the additional assumption on the scalar curvature $\left(0.2^{\prime}\right)$ when $n=3$. In fact, taking $t=1$ and examining the 
dependence of $B_{1}, B_{2}$ on $\beta, c_{0}$ and $A_{g_{k}}$, and using the fact that $A_{g_{k}} \leq A_{0}$ we have

$$
\begin{aligned}
\left(\int|\nabla \varphi|^{6} d V\right)^{1 / 6} & \leq C\left(\beta, c_{0}\right) \tilde{\beta}+C\left(\beta, c_{0}\right)\left(\int|\nabla \varphi|^{2} d V\right)^{\frac{1}{2}} \\
& \leq C\left(\beta, \tilde{\beta}, c_{0}, V_{0}\right)
\end{aligned}
$$

where the last inequality follows from (3.6).

If $n \geq 4$, assume that $p<n$. Then if

$$
t \leq \frac{2 p}{2 N+p(2-N)} \equiv T_{0}
$$

we have (by Hölder)

$$
\|R\|_{\overline{N t+2(1-t)}} \leq C\left(V_{0}, \beta\right)
$$

and conclude

$$
\|\nabla \varphi\|_{N t} \leq \Lambda_{2}\left(\|\nabla \varphi\|_{2 t}+1\right)
$$

where $\Lambda_{2}=\Lambda_{2}\left(V_{0}, \beta, c_{0}, n, \rho\right)$. We now proceed to iterate (3.13) in order to show (3.2).

Let $k$ be the smallest integer such that

$$
q \equiv\left(\frac{2}{N}\right)^{k}\left(N T_{0}\right) \leq N
$$


Then by (3.13),

$$
\begin{aligned}
\|\nabla \varphi\|_{N T_{0}} & \leq \Lambda_{2}\left(1+\|\nabla \varphi\|_{2 T_{0}}\right) \\
& \leq \Lambda_{2}\left(1+\|\nabla \varphi\|_{N\left(\frac{2}{N} T_{0}\right)}\right) \\
& \leq \Lambda_{2}+\Lambda_{2}^{2}\left(1+\|\nabla \varphi\|_{\left(\frac{4}{N^{2}}\right)\left(N T_{0}\right)}\right) \\
& \vdots \\
& \leq\left(\Lambda_{2}+\Lambda_{2}^{2}+\cdots+\Lambda_{2}^{k}\right)+\Lambda_{2}^{k}\|\nabla \varphi\|_{(2 / N)^{k}\left(N T_{0}\right)}^{k}\left(N T_{0}\right) \\
& \leq C\left(\Lambda_{2}\right)+\Lambda_{2}^{k}\|\nabla \varphi\|_{q} .
\end{aligned}
$$

Now letting $t=1$ in (3.13) shows

$$
\begin{aligned}
\|\nabla \varphi\|_{N} & \leq \Lambda_{2}\left(\|\nabla \varphi\|_{2}+1\right) \\
& \leq C\left(\beta, V_{0}, c_{0}\right)
\end{aligned}
$$

(where again we have used (3.6)), while $q \leq N$ implies by Hölder that

$$
\|\nabla \varphi\|_{q} \leq C\left(V_{0}\right)\|\nabla \varphi\|_{N} \leq C\left(V_{0}, \beta, c_{0}\right) .
$$

Combining this with (3.14) we conclude

$$
\|\nabla \varphi\|_{N T_{0}} \leq C\left(V_{0}, \beta, c_{0}\right) .
$$


To see that (3.2) follows from (3.15), recall that $r_{0}=N T_{0}=n p /(n-p)$, and compute:

$$
\begin{aligned}
\int|\nabla \varphi|^{r_{0}} d V & =\int\left|\nabla_{g_{k}}\left(u_{k}^{-1}\right)\right|^{r_{0}} d V_{g_{k}} \\
& =\int\left(\left|\nabla_{0}\left(u_{k}^{-1}\right)\right| u_{k}^{-\frac{2}{n-2}}\right)^{r_{0}} u_{k}^{N} d V_{0} \\
& =\int\left|\nabla_{0} u_{k}\right|^{r_{0}} u_{k}^{-2 r_{0}\left(\frac{n-1}{n-2}\right)+N} d V_{0} \\
& =s_{0}^{-r_{0}} \int\left|\nabla_{0}\left(u_{k}^{-s_{0}}\right)\right|^{r_{0}} d V_{0},
\end{aligned}
$$

where $s_{0}=[p(n+2)-2 n] / p(n-2)$.

If $n=3$, then

$$
\int|\nabla \varphi|^{6} d V=2^{-6} \int \mid \nabla_{0}\left(u_{k}^{-2}\right)^{6} d V_{0} .
$$

We have therefore shown that Proposition 3.2 follows from Proposition 3.3. So in order to conclude Proposition 3.2 (hence by our earlier work, Proposition 3.1), we now proceed to prove Proposition 3.3.

We begin with a lemma.

LEMMA 2.4. Let $(M, h)$ be a compact $n$-dimensional manifold with $n \geq 3$. If $\varphi \in C^{\infty}(M)$, then for any $q \geq 1$ we have

$$
\begin{array}{r}
\int\left|\nabla_{h} \varphi\right|^{q}\left|\nabla_{h}^{2} \varphi\right|^{2} d V_{h} \leq(1+q / 4) \int\left|\nabla_{h} \varphi\right|^{q}\left(\Delta_{h} \varphi\right)^{2} d V_{h} \\
+\int\left|R c_{h}\right|\left|\nabla_{h} \varphi\right|^{q+2} d V_{h} .
\end{array}
$$


Proof. Let $S=\sqrt{\varepsilon+\left|\nabla_{h} \varphi\right|^{2}}$, where $\varepsilon>0$. For tangent vectors $X, Y$ we denote $h(X, Y)=\langle X, Y\rangle$. To simplify notation we drop the subscript $h$, and compute:

$$
\begin{aligned}
\int(\Delta \varphi)^{2} S^{q} d V & =\int(\Delta \varphi)\left(S^{q} \Delta \varphi\right) d V \\
& =-\int\left\langle\nabla \varphi, \nabla\left(S^{q} \Delta \varphi\right)\right\rangle d V \\
& =-\int\left\langle\nabla \varphi, S^{q} \nabla(\Delta \varphi)+q S^{q-1}(\Delta \varphi) \nabla S\right\rangle d V \\
& =-\int S^{q}\langle\nabla \varphi, \nabla(\Delta \varphi)\rangle d V-q \int S^{q-1}(\Delta \varphi)\langle\nabla \varphi, \nabla S\rangle d V
\end{aligned}
$$

Recall the Bochner identity:

$$
\Delta|\nabla \varphi|^{2}=2\left|\nabla^{2} \varphi\right|^{2}+2 R c(\nabla \varphi)+2\langle\nabla \varphi, \nabla(\Delta \varphi)\rangle .
$$

Substituting this into the above equation we have

$$
\begin{aligned}
\int(\Delta \varphi)^{2} S^{q} d V=-\int S^{q}\left\{\frac{1}{2} \Delta|\nabla \varphi|^{2}-\left|\nabla^{2} \varphi\right|^{2}-R c(\nabla \varphi)\right\} d V \\
\quad-q \int S^{q-1}(\Delta \varphi)\langle\nabla \varphi, \nabla S\rangle d V \\
=-\frac{1}{2} \int S^{q} \Delta|\nabla \varphi|^{2} d V+\int S^{q}\left|\nabla^{2} \varphi\right|^{2} d V \\
+\int S^{q} R c(\nabla \varphi) d V-q \int S^{q-1}(\Delta \varphi)\langle\nabla \varphi, \nabla S\rangle d V
\end{aligned}
$$


Now,

$$
\begin{aligned}
\Delta S & =\frac{1}{2} S^{-1} \Delta|\nabla \varphi|^{2}-\left.\left.\frac{1}{4} S^{-3}|\nabla| \nabla \varphi\right|^{2}\right|^{2} \\
& =\frac{1}{2} S^{-1} \Delta|\nabla \varphi|^{2}-\left.S^{-3}|\nabla \varphi|^{2}|\nabla| \nabla \varphi\right|^{2} \\
& =\frac{1}{2} S^{-1} \Delta|\nabla \varphi|^{2}-S^{-1}|\nabla S|^{2},
\end{aligned}
$$

where the last line follows from the fact that

$$
|\nabla S|=\left.\frac{1}{2} S^{-1}|\nabla| \nabla \varphi\right|^{2}\left|=S^{-1}\right| \nabla \varphi|| \nabla \mid \nabla \varphi \| .
$$

We also note

$$
\begin{aligned}
\Delta S^{q+2}= & (q+2) S^{q+1} \Delta S+(q+2)(q+1) S^{q}|\nabla S|^{2} \\
= & (q+2) S^{q+1}\left\{\frac{1}{2} S^{-1} \Delta|\nabla \varphi|^{2}-S^{-1}|\nabla S|^{2}\right\} \\
& \quad+(q+2)(q+1) S^{q}|\nabla S|^{2} \\
& =\frac{1}{2}(q+2) S^{q} \Delta|\nabla \varphi|^{2}+q(q+2) S^{q}|\nabla S|^{2}
\end{aligned}
$$

which implies

$$
-\frac{1}{2} S^{q} \Delta|\nabla \varphi|^{2}=-\frac{1}{(q+2)} \Delta S^{q+2}+q S^{q}|\nabla S|^{2} .
$$

Integrating this expression over $M$ gives

$$
-\frac{1}{2} \int S^{q} \Delta|\nabla \varphi|^{2} d V=q \int S^{q}|\nabla S|^{2} d V
$$


Substituting this back into (3.18) we have

$$
\begin{aligned}
\int(\Delta \varphi)^{2} S^{q} d V= & \int S^{q}\left|\nabla^{2} \varphi\right|^{2} d V+\int S^{q} R c(\nabla \varphi) d V \\
& +q \int S^{q}|\nabla S|^{2} d V-q \int S^{q-1}(\Delta \varphi)\langle\nabla \varphi, \nabla S\rangle d V \\
\Rightarrow \quad \int S^{q}\left|\nabla^{2} \varphi\right|^{2} d V= & \int(\nabla \varphi)^{2} S^{q} d V-\int S^{q} R c(\nabla \varphi) d V \\
& -q \int S^{q}|\nabla S|^{2} d V+q \int\left\langle S^{q / 2-1}(\Delta \varphi) \nabla \varphi, S^{q / 2} \nabla S\right\rangle d V \\
\leq & \int(\Delta \varphi)^{2} S^{q} d V+\int|R c| S^{q+2} \\
& -q \int S^{q}|\nabla S|^{2} d V+q \int S^{q}|\nabla S|^{2} \\
& +\frac{1}{4} q \int S^{q-2}|\nabla \varphi|^{2}(\Delta \varphi)^{2} d V \\
\leq & (1+q / 4) \int(\Delta \varphi)^{2} S^{q} d V+\int|R c| S^{q+2} d V .
\end{aligned}
$$

Letting $\varepsilon \rightarrow 0$ gives (3.17).

To continue the Proof of Proposition 3.3, let $f=|\nabla \varphi|^{t}$ in (3.4). Then

$$
\begin{aligned}
\left(\int|\nabla \varphi|^{N t} d V\right)^{2 / N} \leq & A_{h}\left\{\left.\left.\int|\nabla| \nabla \varphi\right|^{t}\right|^{2} d V+\int|\nabla \varphi|^{2 t} d V\right\} \\
\leq & A_{h}\left\{\left.t^{2} \int|\nabla \varphi|^{2 t-2}|\nabla| \nabla \varphi\right|^{2} d V\right. \\
& \left.\quad+\int|\nabla \varphi|^{2 t} d V\right\} \\
\leq & A_{h}\left\{t^{2} \int|\nabla \varphi|^{2 t-2}\left|\nabla^{2} \varphi\right|^{2} d V+\int|\nabla \varphi|^{2 t} d V\right\} .
\end{aligned}
$$

If we let $q=2(t-1)$ in (3.17) and substitute the resulting expression into (3.18) 
we find

$$
\begin{aligned}
\left(\int|\nabla \varphi|^{N t} d V\right)^{2 / N} \leq & A_{h} t^{2}\left\{(1+(t-1) / 2) \int|\nabla \varphi|^{2 t-2}(\Delta \varphi)^{2} d V\right. \\
& \left.+\int|R c||\nabla \varphi|^{2 t} d V\right\}+A_{h} \int|\nabla \varphi|^{2 t} d V \\
\leq & A_{h} t^{2}(1+t) \int|\nabla \varphi|^{2 t-2}(\Delta \varphi)^{2} d V \\
& A_{h} t^{2} \int|\nabla \varphi|^{2 t}|R c| d V+A_{h} \int|\nabla \varphi|^{2 t} d V
\end{aligned}
$$

As before, let $E_{b}=\left\{x \in M:|R c|_{x} \geq b\right\} ;$ then

$$
\begin{aligned}
\int|\nabla \varphi|^{2 t}|R c| d V \leq & b \int|\nabla \varphi|^{2 t} d V \\
& +b^{-\frac{2}{n}\left(p-\frac{n}{2}\right)}\|R c\|_{p}^{2 p / n}\left(\int|\nabla \varphi|^{N t} d V\right)^{2 / N}
\end{aligned}
$$

Letting $b=\left[2 A_{h} t^{2}\|R c\|_{p}^{2 p / n}\right]^{n /(2 p-n)}$ we have

$$
\begin{aligned}
\left(\int|\nabla \varphi|^{N t} d V\right)^{2 / N} \leq & A_{h} t^{2}(1+t) \int|\nabla \varphi|^{2 t-2}(\Delta \varphi)^{2} d V \\
& +A_{h}\left\{t^{2}\left[2 A_{h} t^{2}\|R c\|_{p}^{2 p / n}\right]^{n /(2 p-n)}+1\right\} \int|\nabla \varphi|^{2 t} d V \\
& +\frac{1}{2}\left(\int|\nabla \varphi|^{N t} d V\right)^{2 / N}
\end{aligned}
$$

and subtracting gives (3.5).

§4. Finding a Lower Bound

In view of Proposition 3.1, we have reduced the proof of the main theorem to establishing a lower bound for the sequence $\left\{u_{k}\right\}$. In this section we will show 
that in many cases, an a priori lower bound for $\left\{u_{k}\right\}$ can be found. In order to do so, it is useful to isolate the following apparently weak property introduced in [CY2]:

Definition. We say that $\left\{u_{k}\right\}$ satisfies $\left(^{*}\right)$ if there is a point $x \in M$, constants $\rho, \varepsilon, C>0$ such that

$$
\int_{B(x, \rho)} u_{k}^{-\varepsilon} d V_{0} \leq C
$$

Surprisingly, $\left({ }^{*}\right)$ is sufficient to ensure a lower bound.

LEMMA 4.1. Suppose $\left\{u_{k}\right\}$ satisfies the hypotheses of Proposition 2.1, and also $\left(^{*}\right)$. Then there are constants $\delta_{0}, C_{0}^{\prime}>0$ such that

$$
\int_{M} u_{k}^{-\delta_{0}} d V_{0} \leq C_{0}^{\prime}
$$

As a consequence, there is a constant $C_{0}>0$ such that

$$
u_{k} \geq C_{0}
$$

Proof. Let $\delta>0$ and multiply the equation for $u_{k}$ by $u_{k}^{-1-2 \delta}$. Then integrating by parts gives

$$
\int\left|\nabla u_{k}^{-\delta}\right|^{2} d V_{0}=-\alpha_{n} \frac{\delta^{2}}{(1+2 \delta)} \int R_{k} u_{k}^{N-2-2 \delta} d V_{0}+\alpha_{n} \delta^{2} \int R_{0} u_{k}^{-2 \delta} d V_{0}
$$

If $\delta>0$ is small enough so that $p(N-2-2 \delta) \leq N$, then by Hölder

$$
\int\left|\nabla u_{k}^{-\delta}\right|^{2} d V_{0} \leq C\left(V_{0}, \beta\right)+\alpha_{n} \delta^{2}\left\|R_{0}\right\|_{\infty} \int u_{k}^{-2 \delta} d V_{0}
$$


Let $\lambda_{1}$ denote the first non-zero eigenvalue of $\Delta_{g_{0}}$. Then by the Rayleigh inequality,

$$
\begin{aligned}
\int u_{k}^{-2 \delta} d V_{0} & \leq\left(\int u_{k}^{-\delta} d V_{0}\right)^{2} /\left(\int d V_{0}\right)+\frac{1}{\lambda_{1}} \int\left|\nabla u_{k}^{-\delta}\right|^{2} d V_{0} \\
& \leq\left(\int u_{k}^{-\delta} d V_{0}\right)^{2} /\left(\int d V_{0}\right) \\
& +\frac{1}{\lambda_{1}}\left\{C+C \delta^{2} \int u_{k}^{-2 \delta} d V_{0}\right\} .
\end{aligned}
$$

If $\delta<\varepsilon$ (where $\varepsilon$ is the exponent in $\left({ }^{*}\right)$ ) we have

$$
\begin{aligned}
\int u_{k}^{-\delta} d V_{0} & =\int_{B_{\rho}} u_{k}^{-\delta} d V_{0}+\int_{B_{\rho}^{c}} u_{k}^{-\delta} d V_{0} \\
& \leq C+\left(\int u_{k}^{-2 \delta} d V_{0}\right)^{\frac{1}{2}}\left(\int_{B_{\rho}^{c}} d V_{0}\right)^{\frac{1}{2}} .
\end{aligned}
$$

This implies that for any $\eta>0$,

$$
\begin{aligned}
\left(\int u_{k}^{-\delta} d V_{0}\right)^{2} /\left(\int d V_{0}\right) & \leq C_{\eta}+ \\
& (1+\eta)\left(\int_{B_{\rho}^{c}} d V_{0} / \int_{M} d V_{0}\right)\left(\int u_{k}^{-2 \delta} d V_{0}\right) .
\end{aligned}
$$

Substituting this into (4.4) gives

$$
\begin{aligned}
& \int u_{k}^{-2 \delta} d V_{0} \leq C_{\eta}+(1+\eta)\left(\int_{B_{\rho}^{c}} d V_{0} / \int_{M} d V_{0}\right)\left(\int u_{k}^{-2 \delta} d V_{0}\right) \\
&+C \delta^{2} \int u_{k}^{-2 \delta} d V_{0}
\end{aligned}
$$


Now choose $\eta=\eta(\rho)>0$ small enough, then $\delta_{0}>0$ small enough so that the last two terms in the RHS of (4.5) can be absorbed into LHS, giving us (4.1).

To see that (4.2) follows from (4.1), let $G(X, \cdot)$ denote the Green's function for $\Delta_{g_{0}}$ with singularity at $X \in M$. Then $G(X, \cdot) \geq 0$ and $\|G(X, \cdot)\|_{t} \leq C_{t}$ for $t<n /(n-2)$. If $0<\delta<\delta_{0}$,

$$
\begin{aligned}
& u_{k}^{-\delta}(X)= \int u_{k}^{-\delta} d V_{0} / \int d V_{0}-\int G(X, \cdot) \Delta\left(u_{k}^{-\delta}\right) d V_{0} \\
& \leq C-\int G(X, \cdot)\left\{-\delta \alpha_{n} R_{k} u_{k}^{N-2-\delta}-\delta \alpha_{n} R_{0} u_{k}^{-\delta}\right. \\
&\left.\quad+\delta(\delta+1) u_{k}^{-2-\delta}\left|\nabla u_{k}\right|^{2}\right\} d V_{0} \\
& \leq C+\delta \alpha_{n} \int G(X, \cdot) R_{k} u_{k}^{N-2-\delta} d V_{0} \\
&+\delta \alpha_{n} \int G(X, \cdot) R_{0} u_{k}^{-\delta} d V_{0}
\end{aligned}
$$

Now $\left\|R_{k} u_{k}^{N-2-\delta}\right\|_{s} \leq C\left(\beta, V_{0}\right)$ with $s=N /(N-2-\delta)>\frac{n}{2}$ by Hölder. This implies

$$
\begin{aligned}
\left|\int G(X, \cdot) R_{k} u_{K}^{N-2-\delta} d V_{0}\right| & \leq\|G(X, \cdot)\|_{s^{\prime}}\left\|R_{k} u_{k}^{N-2-\delta}\right\|_{s} \\
& \leq C
\end{aligned}
$$

where $1 / s+1 / s^{\prime}=1$ and $s^{\prime}<\frac{n}{n-2}$. This gives a bound for the first integral in the RHS of (4.6). But if $\delta>0$ is chosen small enough, we can use Hölder to bound the third term. This gives us (4.2).

There are two cases for which $\left(^{*}\right)$ can be verified relatively easily. Recall $Q=Q\left(M, g_{0}\right)$ denotes the Yamabe constant of $\left(M, g_{0}\right)$. 
LEMMA 4.2. If $Q \leq 0$ and $\left\{u_{k}\right\}$ satisfies the hypothesis of Proposition 2.1, then $\left\{u_{k}\right\}$ satisfies $\left(^{*}\right)$.

Proof. We first observe that if $\varepsilon_{0}>0$ satisfies $p\left(N-2-2 \varepsilon_{0}\right)=N$, then by (4.3) we have

$$
\int\left|\nabla u_{k}\right|^{2} u_{k}^{-2-2 \varepsilon_{0}} d V_{0} \leq C\left(V_{0}, \beta\right)
$$

This follows from the fact that $R_{0} \leq 0$ (because $Q \leq 0$ ) and

$$
\left|\int R_{k} u_{k}^{N-2-2 \varepsilon_{0}} d V_{0}\right| \leq \beta V_{0}^{(p-1) / p}
$$

by Hölder.

Let $\nu$ be any smooth, positive, non-constant function and let $h=\nu^{4 / n-2} g_{0}$. If we let $w_{k}=u_{k} / \nu$, then

$$
\begin{aligned}
L_{h} w_{k} & =L_{\nu^{4 / n-2} g_{0}}\left(u_{k} / \nu\right) \\
& =\nu^{-N+1}\left(L_{g_{0}} u_{k}\right) \\
& =-\alpha_{n} R_{k} w_{k}^{N-1}
\end{aligned}
$$

If $S$ denotes the scalar curvature of $h$, then

$$
\begin{aligned}
L_{h} & =\Delta_{h}-\alpha_{n} S, \\
S & =-\alpha_{n}^{-1} \nu^{-N+1}\left(L_{g_{0}} \nu\right) .
\end{aligned}
$$

Since $\nu$ is non-constant and $\int\left(\Delta_{g_{0}} \nu\right) d V_{0}=0$, there is an open set $U \subset M$ where $\Delta \nu>0$. Choose a point $x \in U$ and a geodesic ball $B=B(x, \rho)$ whose 
closure is in $U$. By scaling $\nu$ if necessary, we may assume

$$
\min _{\bar{B}} \nu \geq 1, \quad \min _{\bar{B}} \Delta \nu \geq 1
$$

Choose a smooth cut-off function $\eta$ whose support is in $U$ and such that $\eta \equiv 1$ on $B$. Then multiplying equation (4.8) by $\eta^{2} w_{k}^{-1-\varepsilon_{0}}$ and integrating by parts gives

$$
\begin{aligned}
& \int\left(\Delta_{h} w_{k}\right) w_{k}^{-1-\varepsilon_{0}} \eta^{2} d V_{h}-\alpha_{n} \int S \eta^{2} w_{k}^{-\varepsilon_{0}} d V_{h} \\
& =-\alpha_{n} \int R_{k} w_{k}^{N-2-\varepsilon_{0}} \eta^{2} d V_{h} \\
& \Rightarrow \quad\left(1+\varepsilon_{0}\right) \int \eta^{2} w_{k}^{-2-\varepsilon_{0}}\left|\nabla_{h} w_{k}\right|^{2} d V_{h}-2 \int \eta w_{k}^{-1-\varepsilon_{0}}\left\langle\nabla_{h} \eta, \nabla_{h} w_{k}\right\rangle_{h} d V_{h} \\
& -\alpha_{n} \int S \eta^{2} w_{k}^{-\varepsilon_{0}} d V_{h}=-\alpha_{n} \int R_{k} w_{k}^{N-2-\varepsilon_{0}} \eta^{2} d V_{h} .
\end{aligned}
$$

Now

$$
\begin{aligned}
\left|\int R_{k} w_{k}^{N-2-\varepsilon_{0}} \eta^{2} d V_{h}\right| & =\left|\int R_{k} u_{k}^{N-2-\varepsilon_{0}} \eta^{2} \nu^{2+\varepsilon_{0}} d V_{0}\right| \\
& \leq C\left(V_{0}, \beta\right) .
\end{aligned}
$$

Also,

$$
\begin{aligned}
\mid \int \eta w_{k}^{-1-\varepsilon_{0}}\left\langle\nabla_{h} \eta,\right. & \left.\nabla_{h} w_{k}\right\rangle_{h} d V_{h} \mid \\
& \leq\left(\int \eta^{2}\left|\nabla_{h} \eta\right|^{2} d V_{h}\right)^{\frac{1}{2}}\left(\int w_{k}^{-2-2 \varepsilon_{0}}\left|\nabla_{h} w_{k}\right|^{2} d V_{h}\right)^{\frac{1}{2}} \\
& \leq C\left(\int u_{k}^{-2-2 \varepsilon_{0}}\left|\nabla u_{k}\right|^{2} \nu^{4+2 \varepsilon_{0}} d V_{0}\right)^{\frac{1}{2}} \\
& \leq C\left(V_{0}, \beta\right)
\end{aligned}
$$


because of (4.7). Hence by (4.11), (4.12), and (4.13) we have

$$
-\alpha_{n} \int S \eta^{2} w_{k}^{-\varepsilon_{0}} d V_{0} \leq C\left(V_{0}, \beta\right)
$$

Now

$$
\begin{aligned}
-\alpha_{n} \int S \eta^{2} w_{k}^{-\varepsilon_{0}} d V_{0} & =-\alpha_{n} \int\left\{-\alpha_{n}^{-1}\left(L_{g_{0}} \nu\right) \nu^{-N+1}\right\} u_{k}^{-\varepsilon_{0}} \eta^{2} \nu^{N+2 \varepsilon_{0}} d V_{0} \\
& =\int\left(L_{g_{0}} \nu\right) u_{k}^{-\varepsilon_{0}} \eta^{2} \nu^{1+2 \varepsilon_{0}} d V_{0} \\
& \left.\geq \int\left(\Delta_{g_{0}} \nu\right) u_{k}^{-\varepsilon_{0}} \eta^{2} \nu^{1+2 \varepsilon_{0}} d V_{0} \text { (because } R_{0} \leq 0\right) \\
& \geq \int_{B} u_{k}^{-\varepsilon_{0}} d V_{0}
\end{aligned}
$$

so $\left\{u_{k}\right\}$ satisfies $\left(^{*}\right)$.

If $W\left(g_{k}\right)$ denotes the Weyl curvature tensor of $g_{k}$, then

$$
W\left(g_{k}\right)=u_{k}^{4 / n-2} W\left(g_{0}\right)
$$

Consequently,

$$
\beta^{p} \geq \int\left|W\left(g_{k}\right)\right|^{p} d V_{g_{k}}=\int u_{k}^{-(N-2)(p-n / 2)}\left|W\left(g_{0}\right)\right|^{p} d V_{0}
$$

Since $p>n / 2$, if $W\left(g_{0}\right) \not \equiv 0$ we immediately have that $\left\{u_{k}\right\}$ satisfies $\left(^{*}\right)$. We have therefore proved

LEMMA 4.3. If $n \geq 4$ and $\left(M, g_{0}\right)$ is not locally conformally flat then $\left\{u_{k}\right\}$ satisfies $\left(^{*}\right)$. 
The last case of interest, and really the model for the problem in the conformal setting, is the sphere $S^{n}$ with the standard round metric $\overline{g_{0}}$. The conformal group of $\left(S^{n}, \overline{g_{0}}\right)$ is not compact, so in general a sequence of metrics may not satisfy $\left(^{*}\right)$ (see [CY3] for a further discussion of this phenomenon). For example, let $\left\{\varphi_{t}\right\}_{t \geq 1}$ denote the 1-parameter family of conformal transformations induced by stereographic projection and dilation which leave the two poles fixed. Then $\left\{\varphi_{t}^{*} \overline{g_{0}}\right\}$ is a family of metrics which satisfy the hypotheses of the theorem (they are all isometric to $\overline{g_{0}}$ ) but do not have a lower (or upper) bound. However, it is this very 1-parameter family which we will use to show that any sequence of metrics which exhibits this degenerate behavior can be pulled back to a bounded one. This idea of "renormalizing" is due to K. Uhlenbeck.

Proposition 4.4. Suppose $\left(M, g_{0}\right)$ is conformally equivalent to $\left(S^{n}, \overline{g_{0}}\right)$, and that $\left\{u_{k}\right\}$ satisfies the hypotheses of Proposition 2.1. Then if $\left\{u_{k}\right\}$ does not satisfy $\left(^{*}\right)$, there are conformal transformations $\left\{T_{k}\right\}$ such that if $T_{k}^{*} g_{k}=\nu_{k}^{4 / n-2} g_{0}$, then $\left\{\nu_{k}\right\}$ satisfies $(*)$.

Proof. Let us assume $\left(M, g_{0}\right)=\left(S^{n}, \overline{g_{0}}\right)$; the more general case of conformal equivalence follows in an obvious way.

Now, if $\left\{u_{k}\right\}$ does not satisfy $\left({ }^{*}\right)$, then $\max u_{k} \rightarrow \infty$ as $k \rightarrow \infty$. After composing with a rotation, assume that the maximum of $u_{k}$ is achieved at the south pole. To make our calculations explicit, we introduce coordinates $(\zeta, \xi)$ with $\xi \in[-1,1], \zeta \in \mathbf{R}^{n}$. Here $\xi$ is the latitudinal variable so that $\xi=-1$ corresponds to the south pole. If $\left\{\varphi_{t}\right\}_{t \geq 1}$ is the 1-parameter family of conformal 
transformations mentioned above, then $\varphi_{t}^{*} \overline{g_{0}}=\psi_{t}^{4 / n-2} \overline{g_{0}}$, where

$$
\psi_{t}(\zeta, \xi)=\left(\frac{(1+\xi)+t^{2}(1-\xi)}{2 t}\right)^{(2-n) / 2}
$$

Hence, if $\varphi_{t}^{*} g_{k}=\left(\nu_{k, t}\right)^{4 / n-2} \overline{g_{0}}$, then

$$
\nu_{k, t}(\zeta, \xi)=\psi_{t}(\zeta, \xi)\left(u_{k} \circ \varphi_{t}\right)(\zeta, \xi)
$$

For each $k$ choose $t_{k}$ so that $\nu_{k}(0,-1)=1$. Then $\nu_{k} \leq 2^{(n-2) / 2}$ on the southern hemisphere. Hence if $\Omega=\left\{(\zeta, \xi) \in S^{n}: \xi \leq-1 / 2\right\}$, then by the Harnack inequality,

$$
1 \leq \max _{\Omega} \nu_{k} \leq C\left(V_{0}, \beta\right) \min _{\Omega} \nu_{k}
$$

This gives us a uniform lower bound on $\Omega$ and implies that $\left\{\nu_{k}\right\}$ satisfies $\left(^{*}\right)$.

\section{§5. The Degenerate Case}

In $\S 4$ we were able to establish an a priori lower bound for $\left\{u_{k}\right\}$ except when $Q>0$ and $\left(M, g_{0}\right)$ is locally conformally flat. In this section we give a straightforward generalization of the argument in [CY2] to higher dimensions in order to show

Proposition 5.1. Suppose $Q>0$ and $\left(M, g_{0}\right)$ is locally conformally flat. If $\left\{u_{k}\right\}$ does not satisfy $\left(^{*}\right)$, then $\left(M, g_{0}\right)$ is conformally equivalent to $\left(S^{n}, \overline{g_{0}}\right)$.

Since the case where $\left(M, g_{0}\right)$ is the standard sphere was treated in $\S 4$, this will complete the proof of the theorem. 
Proof. Since $\left(^{*}\right)$ fails, $u_{k} \rightarrow 0$ in $C^{\alpha}$ on compact subsets of $M-\Sigma$, by Proposition 2.1. Given $r>0$ and small, define

$$
M_{r}=M-\bigcup_{i=1}^{\nu} B\left(x_{1}, r\right)
$$

where $\Sigma=\left\{x_{1}, x_{2}, \ldots, x_{\nu}\right\}$

Now fix $r>0$ small and for each $k$ choose a constant $c_{k}$ so that

$$
c_{k}^{N} \int_{M_{r}} u_{k}^{N} d V_{0}=1
$$

Then $c_{k} \rightarrow \infty$. Letting $\nu_{k}=c_{k} u_{k}$, and $h_{k}=\nu_{k}^{4 / n-2} g_{0}$ we have

$$
\begin{aligned}
\int\left|R c\left(h_{k}\right)\right|^{p} d V_{h_{k}} & =c_{k}^{-(N-2)(p-n / 2)} \int\left|R c\left(g_{k}\right)\right|^{p} d V_{g_{k}} \\
& \rightarrow 0 \text { as } k \rightarrow \infty .
\end{aligned}
$$

Also, $L_{g_{0}} \nu_{k}=\left(-\alpha_{n} R_{k} u_{k}^{N-2}\right) \nu_{k}$, so

$$
\int_{M_{\mathbf{r}}}\left|L_{g_{0}} \nu_{k}\right|^{2 n /(n+2)} d V_{0} \leq C\left(V_{0}, \beta\right) .
$$

In fact,

$$
\begin{aligned}
\int_{M_{r}}\left|L_{g_{0}} \nu_{k}\right| d V_{0} & \leq \alpha_{n} \int_{M_{r}}\left|R_{k}\right| u_{k}^{N-2} \nu_{k} d V_{0} \\
& \leq \alpha_{n} \beta\left(\int_{M_{r}} u_{k}^{N} d V_{0}\right)^{(2 p-n) / n p}\left(\int_{M_{r}} \nu_{k}^{N} d V_{0}\right)^{2 / N} \\
& \rightarrow 0 \text { as } k \rightarrow \infty .
\end{aligned}
$$


Now (5.2) implies that $\left\{\nu_{k}\right\}$ is bounded in $W^{2,2 n /(n+2)}\left(M_{r}\right)$, hence in $W^{\mathbf{1 , 2}}\left(M_{2 r}\right)$. Thus $\left\{\nu_{k}\right\}$ has a subsequene which converges weakly to $\Gamma \in W^{1,2}\left(M_{2 r}\right)$ and satisfies (weakly, hence strongly) $L_{g_{0}} \Gamma=0$ on $M_{2 r}$ (by (5.3)). We wish to verify that $\Gamma$ is strictly positive; that will follow from the strong maximum principal (since $R_{0}>0$ ) once we have shown that $\Gamma \not \equiv 0$ on $M_{2 r}$.

Since $\left\|u_{k}\right\|_{C^{\alpha}\left(M_{r / 2}\right)} \leq C_{r}$, by the Harnack inequality

$$
\max _{M_{r}} u_{k} \leq C_{r}\left(V_{0}, \beta\right) \min _{M_{r}} u_{k}
$$

which implies

$$
\max _{M_{r}} \nu_{k} \leq C_{r} \min _{M_{r}} \nu_{k}
$$

Since

$$
1=\int_{M_{r}} \nu_{k}^{N} d V_{0} \leq\left(\int_{M_{r}} d V_{0}\right)\left(\max _{M_{r}} \nu_{k}\right)^{N}
$$

we have $\nu_{k} \geq C>0$ on $M_{r}$ and hence $\Gamma>0$.

For any $0<r^{\prime}<r$ we may apply the same argument to produce $\Gamma^{\prime}>0$, and as in [CY2] we find that $\Gamma^{\prime}$ is proportional to $\Gamma$ so that by adjusting constants we may assume $\Gamma=\Gamma^{\prime}$. Let $r_{j} \rightarrow 0$ and for each $r_{j}$ repeat the process, then take a diagonal subsequence to produce $\Gamma>0$ which satisfies $L_{g_{0}} \Gamma=0$ on $M-\Sigma$.

By the removable singularities result of [GS], either $\Gamma$ has a pole at some $x_{i} \in \Sigma$ or each element of $\Sigma$ is a removable singularity. In the latter case we would have $L_{g_{0}} \Gamma=0$ on $M$ with $\Gamma$ smooth, a contradiction. Hence there is a non-empty set $\Sigma^{1} \subset \Sigma$ such that $x_{i} \in \Sigma^{1}$ if and only if $\Gamma(x) \sim d\left(x, x_{i}\right)^{2-n}$ near $x_{i}$. Let $X=M-\Sigma^{1}$. 
Now $h=\Gamma^{4 / n-2} g_{0}$ is a complete, flat metric on $X$; this follows from (5.1) and the fact that $\left(M, g_{0}\right)$ is locally conformally flat. By the classification theorem for flat space forms (see [W]), this implies that $(X, h)$ is conformally equivalent to the sphere minus $\left|\Sigma^{1}\right|-1$ points and $\left(M, g_{0}\right)$ is therefore conformally equivalent to $\left(S^{n}, \overline{g_{0}}\right)$.

\section{REFERENCES}

[BPY] Brooks, R., P. Perry, and P. Yang, Isospectral sets of conformally equivalent metrics, Duke Jour. Math., 58 (1989), 131-150.

[CY1] Chang, S.-Y. A., and P. Yang, Compactness of isospectral conformal metrics on $S^{3}$, Comment. Math. Helvetici, 64 (1989), 363-374.

[CY2] Chang, S.-Y. A., and P. Yang, Isospectral conformal metrics on 3-manifolds, Jour. Amer. Math. Society, 3 (1990), 117-145.

[CY3] Chang, S.-Y. A., and P. Yang, The conformal deformation equation and isospectral sets of conformal metrics, Contemporary Math., 101 (1989), $165-178$.

[Cr] Croke, C., Some isoperimetric inequalities and eigenvalue estimates, $A n n$. Scient. Ec. Norm. Sup., 13 (1980), 419-435.

[G] Gilkey, P., Leading terms in the asymptotics of the heat equation, Contemporary Math., 73, (1988), 79-85.

[LP] Lee, J., and T. Parker, The Yamabe problem, Bull. Amer. Math. Soc. (N.S.), 17 (1987), 37-81.

[LF] Lelong-Ferrand, J., Transformations conformers et quasi-conformes des varieties riemanniennes compactes applicationa la demonstration d'une 
conjecture de A. Lichnerowicz, C.R. Acad. Sci. Paris, 269 (1969), 583586.

[MS] McKean, H., and I. Singer, Curvature and the eigenvalues of the Laplacian, J. Diff. Geom., 1 (1967), 43-69.

[OPS1] Osgood, G., R. Phillips, and P. Sarnak, Extremals of determinants of Laplacians, J. Funct. Anal., 80 (1988), 148-211.

[OPS2] Osgood, G., R. Phillips, and P. Sarnak, Compact isospectral sets of surfaces, J. Funct. Anal., 80 (1988), 212-234.

[P] Peters, S., Convergence of Riemannian manifolds, Compositio Math., 62 (1987), 3-16.

[S] Scheen, R., Conformal determination of a Riemannian metric to constant scalar curvature, J. Diff. Geom., 21 (1984), 479-495.

[T] Trudinger, N., Remarks concerning the conformal deformation of Riemannian structures on compact manifolds, Ann. Scuola Norm. Pisa, 22 (1968), 265-274.

[W] Wolf, J., Spaces of constant curvature, McGraw-Hill, New York, 1967.

$[Y]$ Yamabe, H., On a deformation of Riemannian structures on compact manifolds, Osaka Math. J. 12 (1960), 21-37.

[Yg] Yang, D., $L^{p}$ pinching and compactness theorems for compact Riemannian manifolds, preprint. 